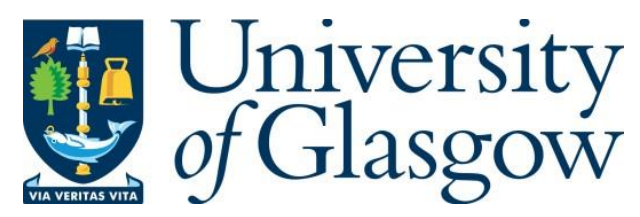

Li, K., Ogden, R. W. and Holzapfel, G. A. (2018) Modeling of fibrous biological tissues with a general invariant that excludes compressed fibers. Journal of the Mechanics and Physics of Solids, 110, pp. 38-53.

There may be differences between this version and the published version. You are advised to consult the publisher's version if you wish to cite from it.

http://eprints.gla.ac.uk/149302/

Deposited on: 6 October 2017

Enlighten - Research publications by members of the University of Glasgow http://eprints.gla.ac.uk 


\title{
Modeling of Fibrous Biological Tissues with a General Invariant that Excludes Compressed Fibers
}

\author{
Kewei $\mathrm{Li}^{1}$, Ray W. Ogden ${ }^{2}$ and Gerhard A. Holzapfel ${ }^{1,3 \dagger}$ \\ ${ }^{1}$ Institute of Biomechanics, Graz University of Technology \\ Stremayrgasse 16-II, 8010 Graz, Austria \\ ${ }^{2}$ School of Mathematics and Statistics, University of Glasgow \\ University Place, Glasgow G12 8SQ, Scotland, UK \\ ${ }^{3}$ Faculty of Engineering Science and Technology,
}

Norwegian University of Science and Technology (NTNU), 7491 Trondheim, Norway

\author{
'J. Mech. Phys. Solids' \\ Accepted September 6, 2017
}

\begin{abstract}
Dispersed collagen fibers in fibrous soft biological tissues have a significant effect on the overall mechanical behavior of the tissues. Constitutive modeling of the detailed structure obtained by using advanced imaging modalities has been investigated extensively in the last decade. In particular, our group has previously proposed a fiber dispersion model based on a generalized structure tensor. However, the fiber tension-compression switch described in that study is unable to exclude compressed fibers within a dispersion and the model requires modification so as to avoid some unphysical effects. In a recent paper we have proposed a method which avoids such problems, but in this present study we introduce an alternative approach by using a new general invariant that only depends on the fibers under tension so that compressed fibers within a dispersion do not contribute to the strain-energy function. We then provide expressions for the associated Cauchy stress and elasticity tensors in a decoupled form. We have also implemented the proposed model in a finite element analysis program and illustrated the implementation with three representative examples: simple tension and compression, simple shear, and unconfined compression on articular cartilage. We have obtained very good agreement with the analytical solutions that are available for the first two examples. The third example shows the efficacy of the fibrous tissue model in a larger scale simulation. For comparison we also provide results for the three examples with the compressed fibers included, and the results are completely different. If the distribution of collagen fibers is such that it is appropriate
\end{abstract}

\footnotetext{
${ }^{\dagger}$ To whom correspondence should be addressed. Email address: holzapfel@tugraz . at
} 
to exclude compressed fibers then such a model should be adopted.

Keywords: Constitutive modeling; fiber dispersion; compressed fiber exclusion; general invariant; articular cartilage

\section{Introduction}

Constitutive models of fibrous soft biological tissues that have been proposed to account for the underlying microstructure have been employed extensively to simulate the mechanical response of the tissues (see, e.g., [1, 2]), and to inform the development of new medical devices [3]. The latest advances in imaging techniques have revealed details of the microstructure of biological tissues such as arterial walls [4-6]. Fiber dispersions have been observed not only in arterial walls but also in articular cartilage [7, 8], carotid arteries [9], the myocardium [10, 11], the pericardium [12], and other tissues. In particular, the knowledge of layer-specific three-dimensional (3D) dispersion of collagen fibers embedded in the ground substance of tissues provides a better understanding of the underlying mechanism of tissue mechanical behavior and facilitates the development of new constitutive models.

The mathematical description of fiber dispersion in a constitutive equation for computational simulations of fibrous tissues poses formidable challenges even with considerable idealizations and simplifications. Since the pioneering work of Lanir [13] on the angular integration (AI) approach for incorporating fiber dispersion in a strain-energy function there have been numerous studies based on this approach; see, e.g., the review article [14] and references therein, in addition to more recent works such as $[15,16]$. Although the physical interpretation of the AI approach is clear and easy to understand, its computational implementation requires numerical integration over a spherical domain at each Gauss point during a finite element analysis, which is computationally expensive. When exclusion of compressed fibers is considered, the AI approach requires even more computational time, which could be reduced by using a highperformance computing cluster [16].

By contrast the generalized structure tensor (GST) approach [17] requires much less computational time, and recently this approach has been shown to be equivalent in predictive power to that of the AI approach [18]. In passing we note that it has recently been brought to our attention that a notion equivalent to our generalized structure tensor was introduced (much) earlier in the context of the rheology of short fiber composites by Advani and Tucker [19]. The GST approach has been used extensively in recent years and is based on a so-called generalized 
structure tensor $\mathbf{H}$ defined by

$$
\mathbf{H}=\frac{1}{4 \pi} \int_{\mathbb{S}^{2}} \rho(\Theta, \Phi) \mathbf{N} \otimes \mathbf{N} \sin \Theta \mathrm{d} \Theta \mathrm{d} \Phi,
$$

where $\Theta$ and $\Phi$ are two spherical polar angles, $\mathbb{S}^{2}=\{(\Theta, \Phi) \mid \Theta \in[0, \pi], \Phi \in[0,2 \pi]\}$ denotes a unit sphere, and the probability density function (PDF) $\rho(\Theta, \Phi)$ represents the relative probability density of fibers at an arbitrary orientation $\mathbf{N}$ around a mean direction $\mathbf{M}$ in the reference configuration of the tissue. The PDF can be determined from imaging data of the fiber distribution in the tissue, and the PDF is normalized according to

$$
\frac{1}{4 \pi} \int_{\mathbb{S}^{2}} \rho(\Theta, \Phi) \sin \Theta \mathrm{d} \Theta \mathrm{d} \Phi=1
$$

In addition, a Green-Lagrange strain-like quantity $E$ was introduced as

$$
E=\frac{1}{4 \pi} \int_{\mathbb{S}^{2}} \rho(\Theta, \Phi) I_{4}(\mathbf{N}) \sin \Theta \mathrm{d} \Theta \mathrm{d} \Phi-1
$$

where $I_{4}=\mathbf{N} \cdot \mathbf{C N}$ is a pseudo-invariant [20], which is equal to the square of the fiber stretch in the direction $\mathbf{N}$, and $\mathbf{C}$ is the right Cauchy-Green tensor. This quantity $E$ was used in the strain-energy function introduced in [17], which is now referred to as the GOH model.

In the original work [17], it was stated that the fibers would contribute to the strain-energy function via $\mathbf{H}$ when the strain in the mean fiber direction $\mathbf{M}$ is positive. However, for computational purposes this condition was implemented as $\bar{E}>0$, where $\bar{E}$ is defined in (3), with $I_{4}$ replaced by its isochoric counterpart $\bar{I}_{4}=\mathbf{N} \cdot \overline{\mathbf{C}} \mathbf{N}$, where $\overline{\mathbf{C}}=(\operatorname{det} \mathbf{C})^{-1 / 3} \mathbf{C}$. In the nonlinear finite element program ABAQUS [21] the GOH model is implemented by using $\bar{E}>0$ for the switch. This leads to continuous stresses and their derivatives, whereas a switch based on the strain in the mean fiber direction may lead to discontinuous stresses and derivatives. When the mean fiber direction in a dispersion is extended then in general some of the fibers in the dispersion will be compressed and such fibers are not excluded by the GOH model. For an incompressible material it is always the case that some fibers are compressed when others are extended and vice versa.

It is not surprising that this rather 'abrupt' treatment leads to a discontinuous stress response as revealed in the recent study [22] since the authors misinterpreted the GOH model. In that study, an equivalent transversely isotropic deformation state was defined that uses squared stretches in the mean fiber direction and an average of the squared stretches of all the fibers in the plane transverse to the mean direction to exclude compressed fibers from a dispersion, and thus a continuous stress response is achieved. However, as the authors mentioned, if both the squared stretches are greater than one, then no exclusion of the compressed fibers is possible. 
Indeed, for simple shear, it is straightforward to show that this tension-compression criterion for dispersed fibers does not exclude all the compressed fibers when the amount of shear is large. Thus, this approach may be only applicable for some (rather) special cases. This motivates the need for a physically realistic switch for the GOH model which avoids discontinuities. It has been stated several times in the literature that it is not possible to exclude compressed fibers within the GOH approach, but this is not the case, as was recently shown in [23].

In this paper we provide an alternative approach to the exclusion of compressed fibers on a quite different basis to the one in [23]. Again we consider $\rho(\Theta, \Phi)$ to satisfy the normalization condition (2) and it follows from (3) that $E=0$ for deformations for which $I_{4} \equiv 1$, i.e. at the boundary between stretched and compressed fibers.

The present study is structured as follows. In Section 2 we describe the form of a new general invariant that excludes fibers under compression. Then, based on this new invariant, we present a new strain-energy function in which the total contribution of all the fibers depends only on the strain energy of the fibers under tension. Next, for the purpose of finite element implementation, in Section 3, we present the continuum mechanical framework of the proposed constitutive model in a decoupled form. Expressions for the Cauchy stress and the elasticity tensors needed for the implementation are also provided. In Section 4, the computational aspects of the constitutive model developed in Section 3 are described.

The theoretical development in Section 3 is independent of the PDF and the strain-energy function of a single fiber. However, for the finite element implementation and for numerical examples, specific forms of the strain-energy function and the PDF are needed and therefore given in this section. To demonstrate the accuracy and efficacy of the proposed fiber dispersion model and its implementation, we present three representative numerical examples by using the finite element based numerical integration scheme from [15]. In particular, in the first example, we compare the numerical and analytical solutions for a unit cube under uniaxial tension or compression in the mean fiber direction. In the second example, we consider simple shear of the same unit cube. The third example deals with an unconfined compression test on a circular cylindrical specimen of articular cartilage. This example is chosen because the majority of the fibers in cartilage are compressed when it is subjected to an unconfined compression test. Thus, the compressive effects of excluding or including compressed fibers can easily be identified. Finally, in Section 5, we summarize the proposed computational modeling framework and discuss possible extensions of the present work. 


\section{A fiber dispersion model based on a general invariant}

For computational simulations, fibrous soft biological tissues are often treated as incompressible, elastic and fiber-reinforced continuum bodies with 3D or 2D fiber dispersions. When layer-specific material properties of the tissue are available, as in [4], then each layer should be treated separately.

Within a fiber dispersion, the fiber orientations $\mathbf{N}$ and $-\mathbf{N}$ represent the same fiber. In the following we therefore confine attention to a unit hemisphere $\mathbb{S}=\{(\Theta, \Phi) \mid \Theta \in[0, \pi], \Phi \in$ $[-\pi / 2, \pi / 2]\}$ instead of $\mathbb{S}^{2}$ since we do not distinguish between $\mathbf{N}$ and $-\mathbf{N}$. Now, in order to exclude the compressed fibers within the dispersion when the material is deformed, we introduce a new general invariant, denoted $I$, which depends only on fibers that are under tension, i.e.

$$
I=\frac{1}{2 \pi} \int_{\Omega(\mathbf{C})} \rho(\Theta, \Phi) f\left(I_{4}\right) \sin \Theta \mathrm{d} \Theta \mathrm{d} \Phi
$$

where $\Omega(\mathbf{C})=\left\{(\Theta, \Phi) \mid \Theta \in[0, \pi], \Phi \in[-\pi / 2, \pi / 2], I_{4}>1\right\}$ represents the deformationdependent domain within $\mathbb{S}$ where fibers are under tension, and $f\left(I_{4}\right)$ denotes a scalar function dependent on the direction of $\mathbf{N}$ and the deformation $\mathbf{C}$. We require $f(1)=0$ and $f^{\prime}(1)=$ 0 , where $f^{\prime}\left(I_{4}\right)=\partial f / \partial I_{4}$, so that $I$ vanishes on the boundary of $\Omega(\mathbf{C})$. We also require $f\left(I_{4}\right)>0$ and $f^{\prime}\left(I_{4}\right)>0$ for $I_{4}>1$. Although we are considering here the hemisphere $\mathbb{S}$, the normalization condition (2), which includes all the fibers, still holds because of symmetry.

Since the fibrous soft biological tissue is considered as an elastic continuum, in this study we assume that there exists a strain-energy function $\Psi(\mathbf{C},\{\mathbf{N}\})$ which depends on the macroscopic deformation of the material through $\mathbf{C}$, the PDF $\rho(\Theta, \Phi)$, and the underlying fiber orientations through $\{\mathbf{N}\}$, where the notation $\{\mathbf{N}\}$ indicates the dependence of $\Psi$ on the distribution of the fiber orientations. Following our previous experimental results [24] and the modeling approach in $[17,25,26]$ we treat the ground substance of the tissue as a neo-Hookean material [20] in terms of the first invariant $I_{1}=\operatorname{tr} \mathbf{C}$ and consider one family of collagen fibers embedded in the ground substance. Thus, the total strain-energy function $\Psi$ per unit reference volume due to the contributions of the ground substance and all the extended fibers reads

$$
\Psi(\mathbf{C},\{\mathbf{N}\})=\Psi_{\mathrm{g}}\left(I_{1}\right)+\Psi_{\mathrm{f}}(I), \quad \Psi_{\mathrm{g}}\left(I_{1}\right)=\frac{\mu}{2}\left(I_{1}-3\right), \quad \Psi_{\mathrm{f}}(I)=\frac{k_{1}}{2 k_{2}}\left[\exp \left(k_{2} I\right)-1\right],
$$

where $\Psi_{\mathrm{g}}$ represents the strain energy stored in the ground substance, $\Psi_{\mathrm{f}}$ is the strain energy accumulated in all the extended fibers, $\mu$ denotes the shear modulus of the ground substance, $k_{1}$ is a positive material parameter with the dimension of stress, $k_{2}$ is a positive dimensionless material parameter, while $I$ is defined by (4). The parameters $k_{1}$ and $k_{2}$ are related to the 
fiber properties. The fiber strain energy $\Psi_{\mathrm{f}}(I)$ increases monotonically with $I$. If an additional fiber family is present in the tissue it can be included additively in a straightforward way, with different parameters $k_{1}$ and $k_{2}$, in general, see the third example in [16].

\section{Continuum mechanical framework}

In this section, the notation and fundamental concepts of nonlinear continuum mechanics are briefly reviewed in order to describe the new fiber dispersion model in a decoupled form. Then, we present expressions for the corresponding Cauchy stress and elasticity tensors, also in decoupled form.

\subsection{Kinematics}

Let us introduce a deformation map $\mathbf{x}=\chi(\mathbf{X})$ that transforms a material point $\mathbf{X}$ in the stressfree reference configuration into a spatial point $\mathbf{x}$ of the material in its deformed configuration. The deformation gradient tensor is defined as $\mathbf{F}(\mathbf{X})=\partial \boldsymbol{\chi}(\mathbf{X}) / \partial \mathbf{X}$. Its determinant $J=\operatorname{det} \mathbf{F}(\mathbf{X})>0$ represents the local volume ratio at point $\mathbf{X}$, and for an incompressible material $J=1$. Let us now introduce the multiplicative decomposition of $\mathbf{F}[27,28]$. Thus, we decouple $\mathbf{F}$ into a volumetric (dilatational) part $J^{1 / 3} \mathbf{I}$ and an isochoric (distortional) part $\overline{\mathbf{F}}=J^{-1 / 3} \mathbf{F}$, with $\operatorname{det} \overline{\mathbf{F}} \equiv 1$. In terms of $\mathbf{F}$ the right Cauchy-Green tensor is given by $\mathbf{C}=\mathbf{F}^{\mathrm{T}} \mathbf{F}$ and its isochoric counterpart is $\overline{\mathbf{C}}=\overline{\mathbf{F}}^{\mathrm{T}} \overline{\mathbf{F}}$ with the corresponding first invariants $I_{1}=\operatorname{tr} \mathbf{C}$ and $\bar{I}_{1}=\operatorname{tr} \overline{\mathbf{C}}$, respectively.

\subsection{Decoupled form of the model}

Following our previous method of excluding fibers under compression in the framework of the AI approach for a general deformation state [15], we first construct a local coordinate system in terms of the normalized eigenvectors $\mathbf{V}_{i}, i=1,2,3$, of $\mathbf{C}$. Within the local coordinate system $\mathbf{V}_{1}, \mathbf{V}_{2}, \mathbf{V}_{3}$ we decompose an arbitrary fiber direction $\mathbf{N}$ (a unit vector) using two spherical polar angles $(\Theta, \Phi)$ according to

$$
\mathbf{N}=\sin \Theta \cos \Phi \mathbf{V}_{1}+\sin \Theta \sin \Phi \mathbf{V}_{2}+\cos \Theta \mathbf{V}_{3}
$$

as shown in Figure 1. We restrict the ranges of the two spherical polar angles to the domain of the unit hemisphere $\mathbb{S}$ so that $\Theta \in[0, \pi]$ and $\Phi \in[-\pi / 2, \pi / 2]$. It is more convenient to describe the boundary of the integration domain $\Omega$ in the local coordinate system $\mathbf{V}_{1}, \mathbf{V}_{2}, \mathbf{V}_{3}$ 


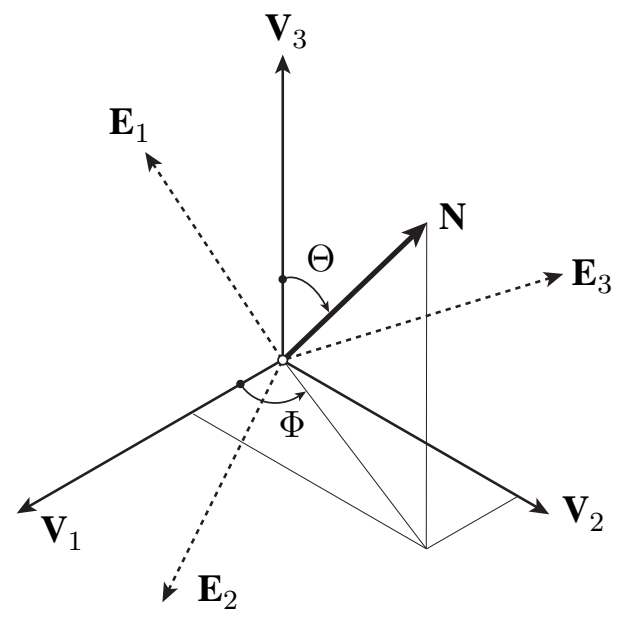

Figure 1: Two spherical polar angles $\Theta \in[0, \pi]$ and $\Phi \in[-\pi / 2, \pi / 2]$ uniquely define an arbitrary fiber direction $\mathbf{N}$ in a local coordinate system constructed by the eigenvectors $\mathbf{V}_{i}$, $i=1,2,3$, of $\mathbf{C}$. The components of $\mathbf{N}$ in the global coordinate system $\mathbf{E}_{i}, i=1,2,3$, can be determined by a rotation tensor $\mathbf{R}$ which relates the coordinate systems $\left(\mathbf{E}_{i}=\mathbf{R}^{\mathrm{T}} \mathbf{V}_{i}\right)$.

instead of the global coordinate system $\mathbf{E}_{1}, \mathbf{E}_{2}, \mathbf{E}_{3}$ [15]; the two coordinate systems are related by $\mathbf{V}_{i}=\mathbf{R E}_{i}, i=1,2,3$, where $\mathbf{R}$ is a rotation tensor dependent on $\mathbf{C}$.

Similarly, we decompose the mean fiber direction $\mathbf{M}$, which is a constant unit vector in the reference configuration, as

$$
\mathbf{M}=\sin \Theta_{\mathrm{M}} \cos \Phi_{\mathrm{M}} \mathbf{V}_{1}+\sin \Theta_{\mathrm{M}} \sin \Phi_{\mathrm{M}} \mathbf{V}_{2}+\cos \Theta_{\mathrm{M}} \mathbf{V}_{3},
$$

where $\Theta_{M}$ and $\Phi_{M}$ can be determined from

$$
\cos \Theta_{\mathrm{M}}=\mathbf{V}_{3} \cdot \mathbf{M}, \quad \tan \Phi_{\mathrm{M}}=\frac{\mathbf{V}_{2} \cdot \mathbf{M}}{\mathbf{V}_{1} \cdot \mathbf{M}} .
$$

On use of (6) the invariant $I_{4}(\mathbf{N})=\mathbf{C}: \mathbf{N} \otimes \mathbf{N}$, where : denotes a double contraction, becomes

$$
\begin{aligned}
I_{4}(\mathbf{N})= & \cos ^{2} \Theta \mathbf{V}_{3} \cdot\left(\mathbf{C} \mathbf{V}_{3}\right)+\sin ^{2} \Theta\left[\cos ^{2} \Phi \mathbf{V}_{1} \cdot\left(\mathbf{C} \mathbf{V}_{1}\right)+\sin ^{2} \Phi \mathbf{V}_{2} \cdot\left(\mathbf{C} \mathbf{V}_{2}\right)\right] \\
& +2 \sin \Theta \cos \Theta\left(\cos \Phi \mathbf{V}_{1}+\sin \Phi \mathbf{V}_{2}\right) \cdot\left(\mathbf{C} \mathbf{V}_{3}\right)+2 \sin ^{2} \Theta \sin \Phi \cos \Phi \mathbf{V}_{1} \cdot\left(\mathbf{C} \mathbf{V}_{2}\right)
\end{aligned}
$$

In terms of its spectral decomposition $\mathbf{C}$ can be written as

$$
\mathbf{C}=\lambda_{1}^{2} \mathbf{V}_{1} \otimes \mathbf{V}_{1}+\lambda_{2}^{2} \mathbf{V}_{2} \otimes \mathbf{V}_{2}+\lambda_{3}^{2} \mathbf{V}_{3} \otimes \mathbf{V}_{3}
$$

where the eigenvalues $\lambda_{i}^{2}, i=1,2,3$, of $\mathbf{C}$ are the squared principal stretches. Hence, (9) reduces to

$$
I_{4}(\Theta, \Phi)=\sin ^{2} \Theta\left(\lambda_{1}^{2} \cos ^{2} \Phi+\lambda_{2}^{2} \sin ^{2} \Phi\right)+\lambda_{3}^{2} \cos ^{2} \Theta
$$


where the argument $\mathbf{N}$ in $I_{4}$ in (9) has been replaced here by $\Theta, \Phi$.

Because $\mathbf{F}$ is decoupled we assume that the total strain-energy function $\Psi$ can be described in terms of an energy contribution $\Psi_{\text {vol }}$ dependent only on $J$, i.e. a purely volumetric contribution, and a contribution $\Psi_{\text {iso }}$ from the isochoric deformation via $\overline{\mathbf{C}}$. Thus [15, 20],

$$
\Psi(\mathbf{C},\{\mathbf{N}\})=\Psi_{\text {vol }}(J)+\Psi_{\text {iso }}(\overline{\mathbf{C}},\{\mathbf{N}\}) .
$$

In addition, we assume that $\Psi_{\text {iso }}$ can be determined by the superposition of the energy contributions $\Psi_{\mathrm{g}}$ from the (non-collagenous) ground substance and $\Psi_{\mathrm{f}}$ from the collagen fibers, i.e.

$$
\Psi_{\text {iso }}=\Psi_{\mathrm{g}}(\overline{\mathbf{C}})+\Psi_{\mathrm{f}}(\overline{\mathbf{C}},\{\mathbf{N}\}) .
$$

Subsequently, we rewrite the general invariant (4) in terms of the modified invariant $\bar{I}_{4}(\mathbf{N})$, i.e.

$$
\bar{I}=\frac{1}{2 \pi} \int_{\Omega(\mathbf{C})} \rho(\Theta, \Phi) f\left(\bar{I}_{4}\right) \sin \Theta \mathrm{d} \Theta \mathrm{d} \Phi .
$$

Because the physical meaning of $\bar{I}_{4}$ is different from that of $I_{4}$ [14], the integration boundary of $\Omega$ is defined by using $I_{4}$ instead of $\bar{I}_{4}$, although there is no difference in the incompressible limit.

Now, when the strain-energy function (5) is expressed in decoupled form the isochoric strain energy of the ground substance depends on the isochoric first invariant $\bar{I}_{1}$, and that of all the extended fibers depends on the isochoric general invariant $\bar{I}$ defined in (14). Hence, the total isochoric strain-energy function $\Psi_{\text {iso }}$ per unit reference volume reads

$$
\Psi_{\text {iso }}=\Psi_{\mathrm{g}}\left(\bar{I}_{1}\right)+\Psi_{\mathrm{f}}(\bar{I}), \quad \Psi_{\mathrm{g}}\left(\bar{I}_{1}\right)=\frac{\mu}{2}\left(\bar{I}_{1}-3\right), \quad \Psi_{\mathrm{f}}(\bar{I})=\frac{k_{1}}{2 k_{2}}\left[\exp \left(k_{2} \bar{I}\right)-1\right] .
$$

Since we are considering an incompressible material, the volumetric strain energy $\Psi_{\text {vol }}$ is not critical here (as it is used as a penalty function), and it is convenient to adopt the form of $\Psi_{\text {vol }}$ used in the FEAP manual [29], i.e.

$$
\Psi_{\mathrm{vol}}=\frac{K}{4}\left(J^{2}-1-2 \ln J\right)
$$

where $K$ is a penalty parameter.

\subsection{Cauchy stress tensor}

We now present the Cauchy stress tensor $\sigma$ for the proposed fiber dispersion model. We first evaluate the fictitious Piola-Kirchhoff stress tensor $\overline{\mathbf{S}}$, which is obtained by differentiating (15) with respect to $\overline{\mathbf{C}} / 2$, i.e.

$$
\overline{\mathbf{S}}=2 \frac{\partial \Psi_{\text {iso }}}{\partial \overline{\mathbf{C}}}=2 \psi_{\mathrm{g}}^{\prime}\left(\bar{I}_{1}\right) \mathbf{I}+2 \psi_{\mathrm{f}}^{\prime}(\bar{I}) \overline{\mathbf{H}}
$$


where $\mathbf{I}$ is the second-order unit tensor, $\psi_{\mathrm{g}}^{\prime}\left(\bar{I}_{1}\right)=\partial \Psi_{\mathrm{g}}\left(\bar{I}_{1}\right) / \partial \bar{I}_{1}=\mu / 2, \psi_{\mathrm{f}}^{\prime}(\bar{I})=\partial \Psi_{\mathrm{f}}(\bar{I}) / \partial \bar{I}=$ $k_{1} \exp \left(k_{2} \bar{I}\right) / 2$, and $\overline{\mathbf{H}}$ is defined as

$$
\overline{\mathbf{H}}=\frac{\partial \bar{I}}{\partial \overline{\mathbf{C}}}=\frac{1}{2 \pi} \int_{\Omega(\mathbf{C})} \rho(\Theta, \Phi) f^{\prime}\left(\bar{I}_{4}\right) \mathbf{N} \otimes \mathbf{N} \sin \Theta \mathrm{d} \Theta \mathrm{d} \Phi,
$$

where $f^{\prime}\left(\bar{I}_{4}\right)=\partial f\left(\bar{I}_{4}\right) / \partial \bar{I}_{4}$. Because the boundary of the integration domain $\Omega(\mathbf{C})$ in the modified general invariant $\bar{I}$ also depends on $\mathbf{C}$, the derivative of $\bar{I}$ with respect to $\overline{\mathbf{C}}$ should, in general, include an integral over the boundary of $\Omega(\mathbf{C})$. This second integral vanishes since $f$ vanishes on the boundary in the incompressible limit. A simple derivation of (18) by using the general Leibniz integral rule and (14) is given in Appendix A for the incompressible limit. A push-forward operation on $\overline{\mathbf{S}}$ with $\overline{\mathbf{F}}$ to the current configuration yields the fictitious Cauchy stress tensor $\bar{\sigma}$ as

$$
\overline{\boldsymbol{\sigma}}=J^{-1} \overline{\mathbf{F}} \overline{\mathbf{S}} \overline{\mathbf{F}}^{\mathrm{T}}=J^{-1}\left[2 \psi_{\mathrm{g}}^{\prime}\left(\bar{I}_{1}\right) \overline{\mathbf{b}}+2 \psi_{\mathrm{f}}^{\prime}(\bar{I}) \overline{\mathbf{h}}\right]
$$

where $\overline{\mathbf{b}}=\overline{\mathbf{F}} \overline{\mathbf{F}}^{\mathrm{T}}$ is the modified left Cauchy-Green tensor, and $\overline{\mathbf{h}}=\overline{\mathbf{F}} \overline{\mathbf{H}} \overline{\mathbf{F}}^{\mathrm{T}}$, the counterpart of $\overline{\mathbf{H}}$ in the Eulerian description, i.e.

$$
\overline{\mathbf{h}}=\frac{1}{2 \pi} \int_{\Omega(\mathbf{C})} \rho(\Theta, \Phi) f^{\prime}\left(\bar{I}_{4}\right) \overline{\mathbf{n}} \otimes \overline{\mathbf{n}} \sin \Theta \mathrm{d} \Theta \mathrm{d} \Phi,
$$

where $\overline{\mathbf{n}}=\overline{\mathbf{F}} \mathbf{N}$. The isochoric part of the Cauchy stress tensor $\boldsymbol{\sigma}_{\text {iso }}$ is then determined as $\boldsymbol{\sigma}_{\text {iso }}=\mathbb{P}: \overline{\boldsymbol{\sigma}}$, where $\mathbb{P}=\mathbb{I}-\frac{1}{3} \mathbf{I} \otimes \mathbf{I}$ is the Eulerian projection tensor [20], and $\mathbb{I}$ is the symmetric fourth-order unit tensor with components $(\mathbb{I})_{a b c d}=\frac{1}{2}\left(\delta_{a c} \delta_{b d}+\delta_{a d} \delta_{b c}\right)$. Finally, the total Cauchy stress tensor is given by $\boldsymbol{\sigma}=\boldsymbol{\sigma}_{\mathrm{vol}}+\boldsymbol{\sigma}_{\text {iso }}$, where the volumetric Cauchy stress tensor $\boldsymbol{\sigma}_{\mathrm{vol}}$ is straightforward to derive [20].

\subsection{Elasticity tensor}

For the computational implementation the elasticity tensor $\mathbb{C}$ in the Eulerian description is also required. Thus, we start with the derivation of the fictitious elasticity tensor $\overline{\mathbb{C}}$ in the Lagrangian description, which is obtained as [20]

$$
\overline{\mathbb{C}}=2 J^{-4 / 3} \frac{\partial \overline{\mathbf{S}}}{\partial \overline{\mathbf{C}}}=4 J^{-4 / 3} \psi_{\mathrm{g}}^{\prime \prime}\left(\bar{I}_{1}\right) \mathbf{I} \otimes \mathbf{I}+4 J^{-4 / 3} \psi_{\mathrm{f}}^{\prime \prime}(\bar{I}) \overline{\mathbf{H}} \otimes \overline{\mathbf{H}}+4 J^{-4 / 3} \psi_{\mathrm{f}}^{\prime}(\bar{I}) \overline{\mathbb{H}}
$$

where

$$
\psi_{\mathrm{g}}^{\prime \prime}\left(\bar{I}_{1}\right)=\frac{\partial^{2} \Psi_{\mathrm{g}}\left(\bar{I}_{1}\right)}{\partial \bar{I}_{1} \partial \bar{I}_{1}}, \quad \psi_{\mathrm{f}}^{\prime \prime}(\bar{I})=\frac{\partial^{2} \Psi_{\mathrm{f}}(\bar{I})}{\partial \bar{I} \partial \bar{I}}
$$


and the fourth-order tensor $\overline{\mathbb{H}}$ is defined as,

$$
\overline{\mathbb{H}}=\frac{\partial \overline{\mathbf{H}}}{\partial \overline{\mathbf{C}}}=\frac{1}{2 \pi} \int_{\Omega(\mathbf{C})} \rho(\Theta, \Phi) f^{\prime \prime}\left(\bar{I}_{4}\right) \mathbf{N} \otimes \mathbf{N} \otimes \mathbf{N} \otimes \mathbf{N} \sin \Theta \mathrm{d} \Theta \mathrm{d} \Phi,
$$

in which $f^{\prime \prime}\left(\bar{I}_{4}\right)=\partial f^{2}\left(\bar{I}_{4}\right) / \partial \bar{I}_{4} \partial \bar{I}_{4}$. Because $\psi_{\mathrm{g}}^{\prime \prime}\left(\bar{I}_{1}\right)=0$ for the neo-Hookean material model, the $\mathbf{I} \otimes \mathbf{I}$ term in $(21)_{2}$ vanishes. A push-forward operation of $\overline{\mathbb{C}}$ with $\mathbf{F}$ and the Piola transform yields the fictitious elasticity tensor in the Eulerian description, i.e.

$$
\overline{\mathbb{C}}=4 J^{-1} \psi_{\mathrm{f}}^{\prime \prime}(\bar{I}) \overline{\mathbf{h}} \otimes \overline{\mathbf{h}}+4 J^{-1} \psi_{\mathrm{f}}^{\prime}(\bar{I}) \overline{\mathbb{H}},
$$

where $\overline{\mathbb{H}}$ is the Eulerian version of $\overline{\mathbb{H}}$ given by

$$
\overline{\mathbb{H}}=\frac{1}{2 \pi} \int_{\Omega(\mathbf{C})} \rho(\Theta, \Phi) f^{\prime \prime}\left(\bar{I}_{4}\right) \overline{\mathbf{n}} \otimes \overline{\mathbf{n}} \otimes \overline{\mathbf{n}} \otimes \overline{\mathbf{n}} \sin \Theta \mathrm{d} \Theta \mathrm{d} \Phi .
$$

Finally, with (24) we obtain the resulting isochoric part of the elasticity tensor in the Eulerian description, i.e. [20]

$$
\mathbb{C}_{\text {iso }}=\mathbb{P}: \overline{\mathbb{C}}: \mathbb{P}+\frac{2}{3} \operatorname{tr}(\overline{\boldsymbol{\sigma}}) \mathbb{P}-\frac{2}{3}\left(\boldsymbol{\sigma}_{\text {iso }} \otimes \mathbf{I}+\mathbf{I} \otimes \boldsymbol{\sigma}_{\text {iso }}\right) .
$$

Then, the total elasticity tensor in the Eulerian description is obtained as $\mathbb{C}=\mathbb{C}_{\mathrm{vol}}+\mathbb{C}_{\text {iso }}$, where the volumetric part $\mathbb{C}_{\mathrm{vol}}$, as for $\boldsymbol{\sigma}_{\mathrm{vol}}$, is straightforward to derive [20].

\section{Computational aspects and representative examples}

We have implemented the general-invariant-based fiber dispersion model (15) in the general purpose finite element analysis program FEAP [29] at the integration point level. Here, for illustration of the method, we simply adopt the quadratic form of $f\left(\bar{I}_{4}\right)$ given by

$$
f\left(\bar{I}_{4}\right)=\left(\bar{I}_{4}-1\right)^{2}
$$

which satisfies the requirements $f(1)=f^{\prime}(1)=0, f\left(I_{4}\right)>0$ and $f^{\prime}\left(I_{4}\right)>0$ for $\bar{I}_{4}>1$.

For purposes of illustration, in this study, we consider a simple case of fiber dispersion in $3 \mathrm{D}$, namely a fiber dispersion which is rotationally symmetric around a mean direction, because the main goal here is to investigate the influence on the mechanical response of excluding compressed fibers from a dispersion. We have therefore adopted a rotationally symmetric fiber dispersion described by the von Mises distribution as

$$
\rho(\Theta, \Phi)=4 \sqrt{\frac{b}{2 \pi}} \frac{\exp \left[2 b(\mathbf{N} \cdot \mathbf{M})^{2}\right]}{\operatorname{erfi}(\sqrt{2 b})},
$$


where $b$ represents a concentration parameter measuring how closely the fibers are distributed around the mean direction $\mathbf{M}$, and $\operatorname{erfi}(x)=-\mathrm{i} \operatorname{erf}(\mathrm{i} x)$ denotes the imaginary error function with the error function $\operatorname{erf}(x)$ defined by

$$
\operatorname{erf}(x)=\frac{2}{\sqrt{\pi}} \int_{0}^{x} \exp \left(-\xi^{2}\right) \mathrm{d} \xi
$$

On substitution of (27) and (28) into the isochoric Cauchy stress tensor $\boldsymbol{\sigma}_{\text {iso }}$ and the Eulerian fictitious elasticity tensor (24), the specific forms of the Cauchy stress and Eulerian elasticity tensors can be obtained. Then, following the method described in [15], we have chosen a finite element based multi-dimensional numerical integration scheme for the evaluation of the double integrals in the Cauchy stress and elasticity tensors [30, 31]. A general guideline for implementation of the proposed fiber dispersion model is shown in the accompanying box (Algorithm 1).

In the following we present three representative examples in order to illustrate the performance and computational implementation of the proposed constitutive model (15). Specifically, simple tension and compression tests on a unit cube in the mean fiber direction, simple shear of the same unit cube, and an unconfined compression test on a cylindrical specimen of articular cartilage cylinder. For each example we assume the material to be incompressible. To enforce the incompressibility condition, we adopt the augmented Lagrangian method [33] in FEAP [29]. In each of the three examples, the geometry of the finite element model was discretized with 8-node hexahedral mixed Q1/P0 elements, and the problems were then solved by using the Newton-Raphson method. The finite element solutions of the first two examples are compared with analytical solutions obtained by using either MATLAB [34] or MATHEMATICA [35]. Due to the non-homogeneous stress distribution, an analytical solution for the last example is not available for comparison. 


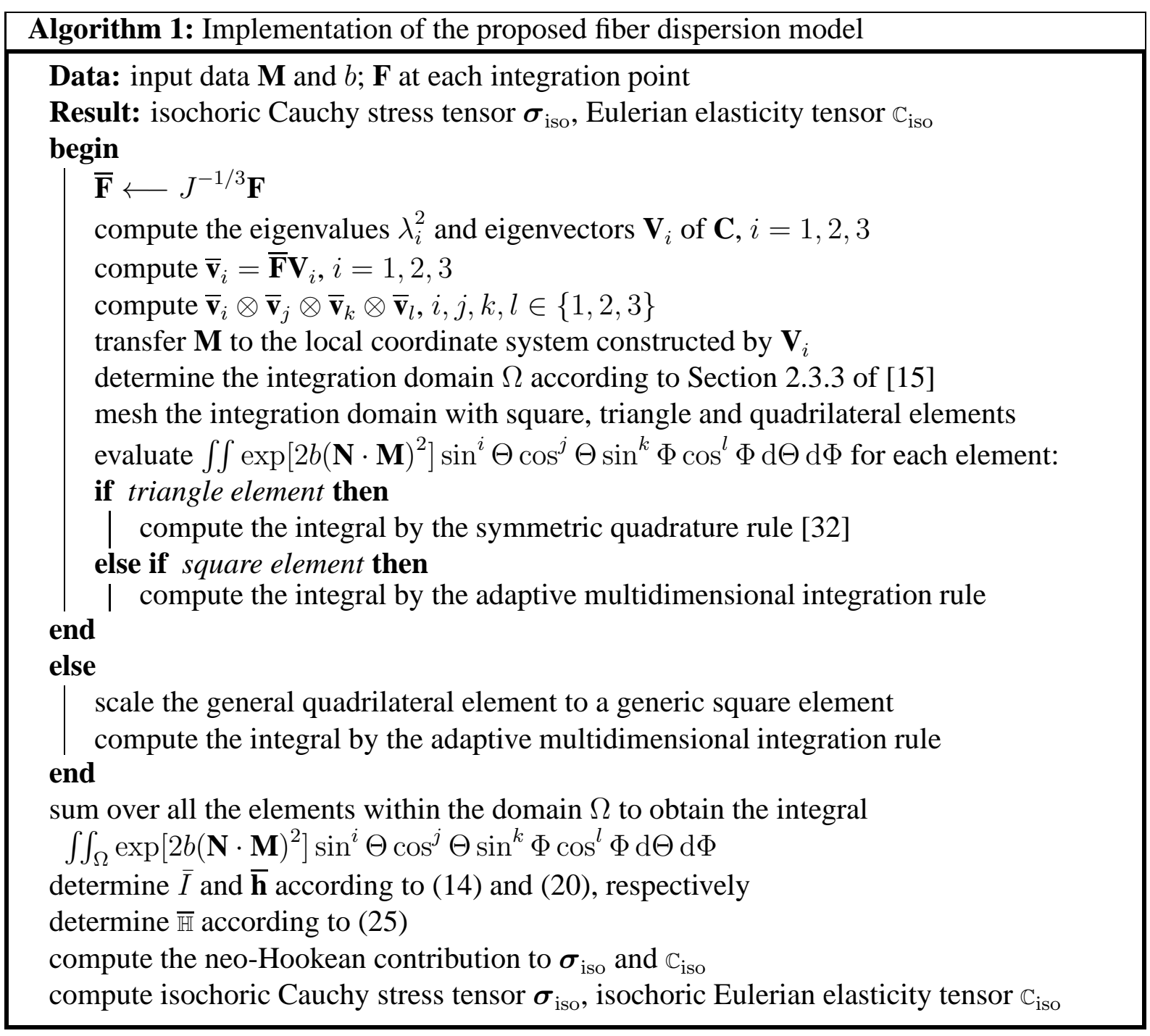

\subsection{Simple tension and compression}

In this first example we consider simple tension and compression in the mean fiber direction $\mathbf{M}=\mathbf{E}_{3}$ of a unit cube composed of one element. We assume that the cube consists of one family of fibers with the PDF given by (28). The cube is aligned with the Cartesian basis vectors $\mathbf{E}_{1}, \mathbf{E}_{2}$ and $\mathbf{E}_{3}$ (see an undeformed cross-section of the cube defined by solid lines in Figure 2) and its dimensions are $1 \times 1 \times 1 \mathrm{~mm}$. A displacement is applied to the top face of the cube, and four nodes at the bottom face are constrained in the $\mathbf{E}_{3}$ direction. To eliminate rigid body translation, the node at $(0,0,0)$ on the bottom face of the cube is constrained also in the $\mathbf{E}_{1}$ and $\mathbf{E}_{2}$ directions; to further prevent rigid body rotation about the $\mathbf{E}_{3}$ direction, the node at $(1,0,0)$ is constrained in the $\mathbf{E}_{2}$ direction, see Figure 2. The resulting deformation under uniaxial tension and compression is homogeneous, and the matrix representations of the deformation gradient 


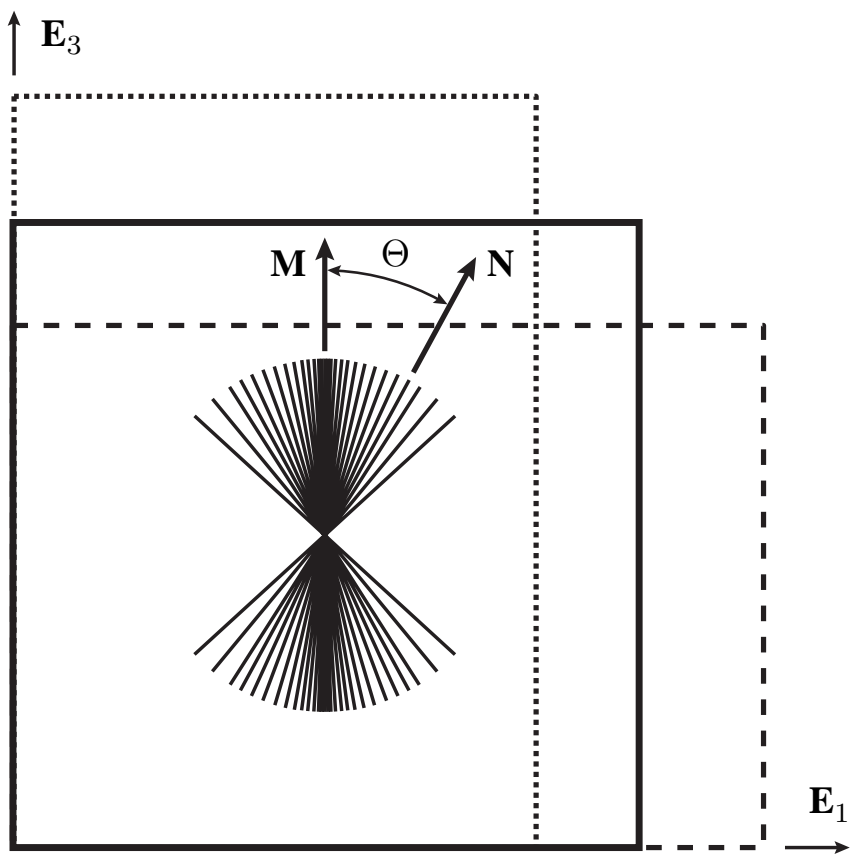

Figure 2: Cross-section of a unit cube under simple tension and compression. The solid lines refer to the reference configuration of the cube cross-section, and the dotted and dashed lines show the deformed configurations of the cube when it is under tension and compression in the $\mathbf{E}_{3}$ direction, respectively. Within a rotationally symmetric fiber dispersion about the mean direction $\mathbf{M}=\mathbf{E}_{3}$, a unit vector $\mathbf{N}$ represents an arbitrary fiber direction.

and the Cauchy-Green tensors are written as

$$
[\mathbf{F}]=\operatorname{diag}\left[\lambda^{-1 / 2}, \lambda^{-1 / 2}, \lambda\right], \quad[\mathbf{b}]=[\mathbf{C}]=\operatorname{diag}\left[\lambda^{-1}, \lambda^{-1}, \lambda^{2}\right],
$$

where $\lambda$ is the principal stretch in the $\mathbf{E}_{3}$ direction. Because the eigenvectors of the right Cauchy-Green tensor coincide with the Cartesian basis vectors, we can simply decompose $\mathbf{N}$ in terms of the Cartesian basis vectors $\mathbf{E}_{i}, i=1,2,3$, as

$$
\mathbf{N}=\sin \Theta \cos \Phi \mathbf{E}_{1}+\sin \Theta \sin \Phi \mathbf{E}_{2}+\cos \Theta \mathbf{E}_{3},
$$

noting, with reference to Figure 1, that $\mathbf{E}_{1}, \mathbf{E}_{2}, \mathbf{E}_{3}$ are principal axes. Thus, with $I_{4}=\mathbf{C}: \mathbf{N} \otimes \mathbf{N}$, from (30) and (31) we obtain

$$
I_{4}(\Theta)=\lambda^{2} \cos ^{2} \Theta+\lambda^{-1} \sin ^{2} \Theta,
$$

which is independent of $\Phi$, the PDF in (28) specializes to

$$
\rho(\Theta)=4 \sqrt{\frac{b}{2 \pi}} \frac{\exp \left(2 b \cos ^{2} \Theta\right)}{\operatorname{erfi}(\sqrt{2 b})},
$$


and the normalization condition (2) reduces to

$$
\int_{0}^{\pi / 2} \rho(\Theta) \sin \Theta \mathrm{d} \Theta=1
$$

To determine the general invariant $I$ for this special case, we first compute the boundary of the integration domain $\Omega$ defined by $I_{4}=1$, which is governed by the equation

$$
\tan \Theta_{\mathrm{c}}=\sqrt{\lambda(\lambda+1)}
$$

where $\Theta_{c} \in\{\Theta \mid 0<\Theta<\pi / 2\}$ denotes the critical angle at which the fiber stretch is one. In $3 D, \Theta=\Theta_{c}$ represents a circle on the hemisphere $\mathbb{S}$. With (27), (32) and (33) the general invariant (4) becomes

$$
I=\left(\lambda^{2}-1\right)^{2} s_{1}+2\left(\lambda^{2}-1\right)\left(\lambda^{-1}-\lambda^{2}\right) s_{3}+\left(\lambda^{-1}-\lambda^{2}\right)^{2} s_{5},
$$

where

$$
s_{1}=\int_{0}^{\Theta_{c}} \rho(\Theta) \sin \Theta \mathrm{d} \Theta, \quad s_{3}=\int_{0}^{\Theta_{c}} \rho(\Theta) \sin ^{3} \Theta \mathrm{d} \Theta, \quad s_{5}=\int_{0}^{\Theta_{c}} \rho(\Theta) \sin ^{5} \Theta \mathrm{d} \Theta .
$$

Now we can evaluate the Cauchy stress tensor $\sigma$ using the specific form (36) of the general invariant $I$, i.e.

$$
\boldsymbol{\sigma}=\mu \mathbf{b}+k_{1} \exp \left(k_{2} I\right) \mathbf{h}-p \mathbf{I},
$$

where $p$ is a Lagrange multiplier and $\mathbf{h}$ is given by

$$
\mathbf{h}=\frac{1}{\pi} \int_{\Omega} \rho(\Theta)\left(I_{4}-1\right) \mathbf{n} \otimes \mathbf{n} \sin \Theta \mathrm{d} \Theta \mathrm{d} \Phi,
$$

where $\Omega=\left\{(\Theta, \Phi) \mid \Theta \in\left[0, \Theta_{c}\right], \Phi \in[0,2 \pi]\right\}, \mathbf{n}=\mathbf{F N}$, and the overbars have been omitted since we are considering an incompressible material here. The uniaxial Cauchy stress $\sigma \equiv \sigma_{33}$ is

$$
\sigma=\mu \lambda^{2}+2 k_{1} \exp \left(k_{2} I\right) \lambda^{2} \alpha-p
$$

where $\alpha$ is defined as

$$
\begin{aligned}
\alpha & =\int_{0}^{\Theta_{c}} \rho(\Theta)\left(I_{4}-1\right) \sin \Theta \cos ^{2} \Theta \mathrm{d} \Theta \\
& =\left(\lambda^{2}-1\right) s_{1}+\left(\lambda^{-1}-2 \lambda^{2}+1\right) s_{3}-\left(\lambda^{-1}-\lambda^{2}\right) s_{5} .
\end{aligned}
$$

Since we are considering uniaxial tension and compression $\left(\sigma_{11}=\sigma_{22}=0\right)$, the Lagrange multiplier is given by

$$
p=\mu \lambda^{-1}+k_{1} \exp \left(k_{2} I\right) \lambda^{-1} \beta, \quad \beta=\left(\lambda^{2}-1\right) s_{3}+\left(\lambda^{-1}-\lambda^{2}\right) s_{5} .
$$




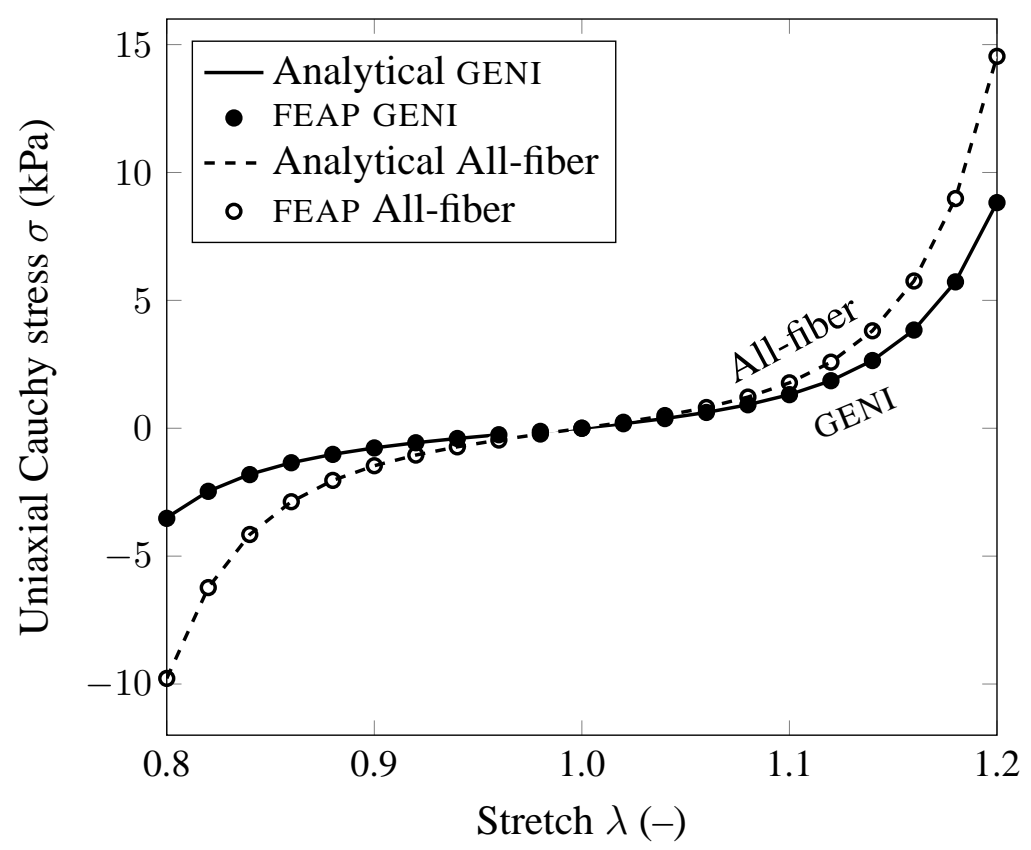

Figure 3: Comparison of the analytical solution obtained with MATLAB and the finite element result obtained with FEAP for the uniaxial Cauchy stress $\sigma$ versus the stretch $\lambda$ for simple tension and compression tests on a unit cube with the parameters $\mu=1.0 \mathrm{kPa}, k_{1}=10.0 \mathrm{kPa}, k_{2}=$ 50.0 , and $b=0.1$. The solid curve and dots represent the material response with compressed fibers excluded (GENI model); the dashed curve and open circles are the results for the case with all fibers included (All-fiber model).

On elimination of $p, \sigma$ becomes

$$
\sigma=\mu\left(\lambda^{2}-\lambda^{-1}\right)+k_{1}\left(2 \lambda^{2} \alpha-\lambda^{-1} \beta\right) \exp \left(k_{2} I\right)
$$

where $\alpha, \beta$ and $I$ depend on $\lambda$ and $\Theta_{\mathrm{c}}$, which itself depends on $\lambda$ via (35).

This equation was implemented in MATLAB [34], and we adopted the built-in adaptive Gauss-Kronrod quadrature method (quadgk) for the evaluation of the integrals in the coefficients $\alpha$ and $\beta$ in (41) and (42) $)_{2}$, respectively. With the parameters $\mu=1.0 \mathrm{kPa}, k_{1}=10.0 \mathrm{kPa}$, $k_{2}=50.0$, and $b=0.1$, the uniaxial Cauchy stress $\sigma$ versus the stretch $\lambda$ is plotted as a solid curve in Figure 3. For comparison with the finite element solution obtained with FEAP [29], we have plotted the numerical results as solid dots in the figure. Clearly, there is a very good agreement between the computational and analytical solutions. Also shown in the figure are the computational and analytical solutions for the case in which the coefficients $\alpha$ and $\beta$ are evaluated numerically over the entire hemisphere $\mathbb{S}$ instead of $\Omega$ (dashed curve and open circles in Figure 3). In the following, we refer to this method as 'All-fiber model' in contrast to the 
general invariant model $I$, abbreviated as 'GENI model', in which compressed fibers are excluded. As shown, the difference between the two methods increases gradually with increasing load for both tension and compression. In addition, the absolute values of $\sigma$ for each test with the All-fiber model (including compressed fibers) are larger than for the GENI model (excluding compressed fibers). This may be explained by the fact that in the GENI model the strain energy of the compressed fibers is excluded from the total strain-energy function, and this reduces the magnitude of the stress.

\subsection{Simple shear}

In this second example we subject the same unit cube to a simple shear deformation in order to test the performance of the proposed constitutive model in predicting shear stress. In order to demonstrate the significant differences between the predictions of the GENI model and the Allfiber model, the mean fiber direction $\mathbf{M}$ is aligned at $135^{\circ}$ in the clockwise direction from the $\mathbf{E}_{3}$ axis in the $\left(\mathbf{E}_{1}, \mathbf{E}_{3}\right)$ plane in the reference configuration, as illustrated by the cross-section of the cube in Figure 4. For the simple shear deformation, we constrained the four nodes on the bottom face of the cube in all three translational degrees of freedom, and then applied a horizontal displacement in the $\mathbf{E}_{1}$ direction on the top face.

We write the matrix forms of the deformation gradient $\mathbf{F}$ and the right Cauchy-Green tensor $\mathbf{C}$ as

$$
[\mathbf{F}]=\left[\begin{array}{ccc}
1 & 0 & c \\
0 & 1 & 0 \\
0 & 0 & 1
\end{array}\right], \quad[\mathbf{C}]=\left[\begin{array}{ccc}
1 & 0 & c \\
0 & 1 & 0 \\
c & 0 & \left(1+c^{2}\right)
\end{array}\right],
$$

and we decompose an arbitrary fiber direction $\mathbf{N}$ as in (31). Then, the squared fiber stretch $I_{4}$ in the direction $\mathbf{N}$ reads

$$
I_{4}(\Theta, \Phi)=1+c^{2} \cos ^{2} \Theta+c \sin 2 \Theta \cos \Phi .
$$

The amount of shear $c$ is assumed to be positive, so the integration domain $\Omega$ is then obtained from the inequality

$$
c \cos ^{2} \Theta+\sin 2 \Theta \cos \Phi>0 .
$$

The general invariant $I$ now specializes to

$$
I=\frac{c^{4}}{2 \pi} t_{1}+\frac{2 c^{3}}{\pi} t_{2}+\frac{2 c^{2}}{\pi} t_{3},
$$




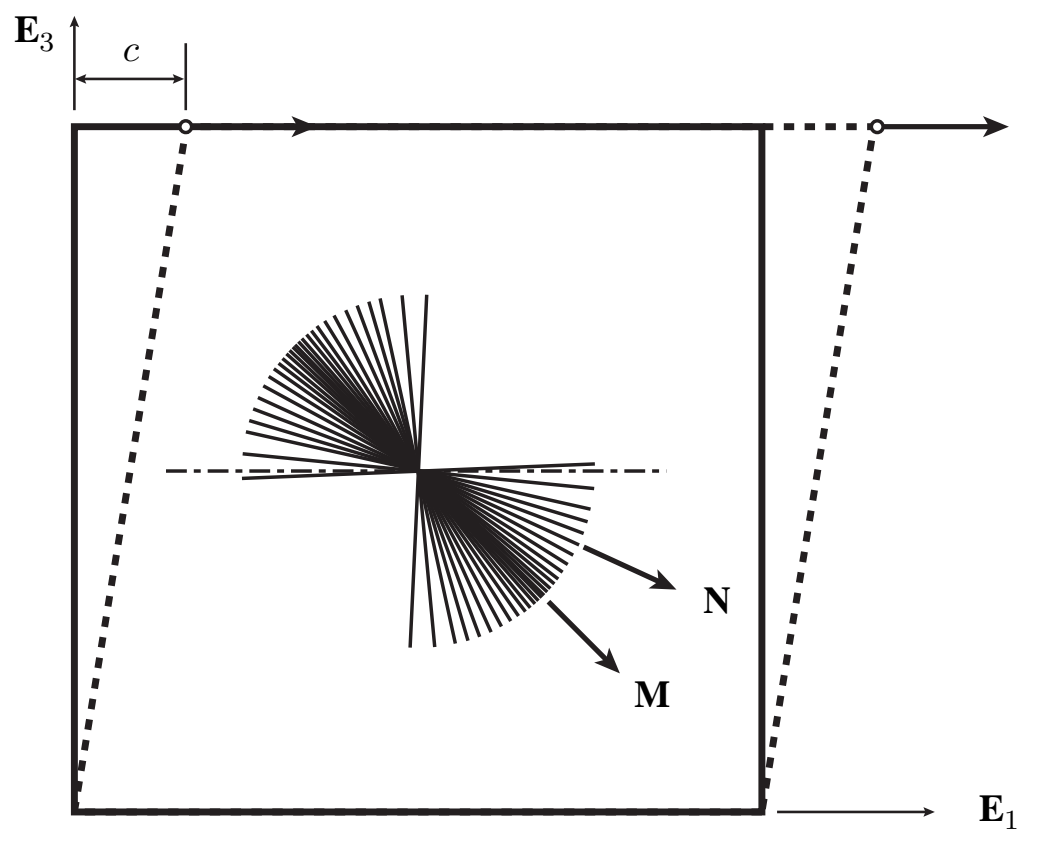

Figure 4: Cross-section of a unit cube subjected to simple shear in the $\left(\mathbf{E}_{1}, \mathbf{E}_{3}\right)$ plane. The solid lines refer to the reference configuration of the cube cross-section, and the dashed lines correspond to the deformed configuration of the cube for an amount of shear $c$. The mean fiber direction $\mathbf{M}$ is aligned at $135^{\circ}$ measured clockwise from the $\mathbf{E}_{3}$ axis. A rotationally symmetric fiber dispersion about $\mathbf{M}$ is assumed. The vector $\mathbf{N}$ represents an arbitrary fiber direction within the dispersion.

where

$$
\begin{aligned}
& t_{1}=\int_{\Omega} \rho(\Theta, \Phi) \sin \Theta \cos ^{4} \Theta \mathrm{d} \Theta \mathrm{d} \Phi, \\
& t_{2}=\int_{\Omega} \rho(\Theta, \Phi) \sin ^{2} \Theta \cos ^{3} \Theta \cos \Phi \mathrm{d} \Theta \mathrm{d} \Phi, \\
& t_{3}=\int_{\Omega} \rho(\Theta, \Phi) \sin ^{3} \Theta \cos ^{2} \Theta \cos ^{2} \Phi \mathrm{d} \Theta \mathrm{d} \Phi .
\end{aligned}
$$

Similarly to the preceding section, it is straightforward to derive the Cauchy stress components with respect to the Cartesian basis vectors. In particular, to evaluate the shear stress component $\sigma_{13}$, we first substitute $\mathbf{n}=\mathbf{F N}$ into the expression for $\mathbf{h}$ in (39), and then substitute $\mathbf{h}$ into (38), yielding

$$
\sigma_{13}=\mu c+\frac{k_{1}}{\pi} \exp \left(k_{2} I\right)\left(c^{3} t_{1}+3 c^{2} t_{2}+2 c t_{3}\right) .
$$

The normal components of the Cauchy stress induced by the simple shear deformation can be calculated by the method described in our previous work [15], but are not needed here. Similarly to that study, we implemented the equation (49) in MATHEMATICA [35] by using the 


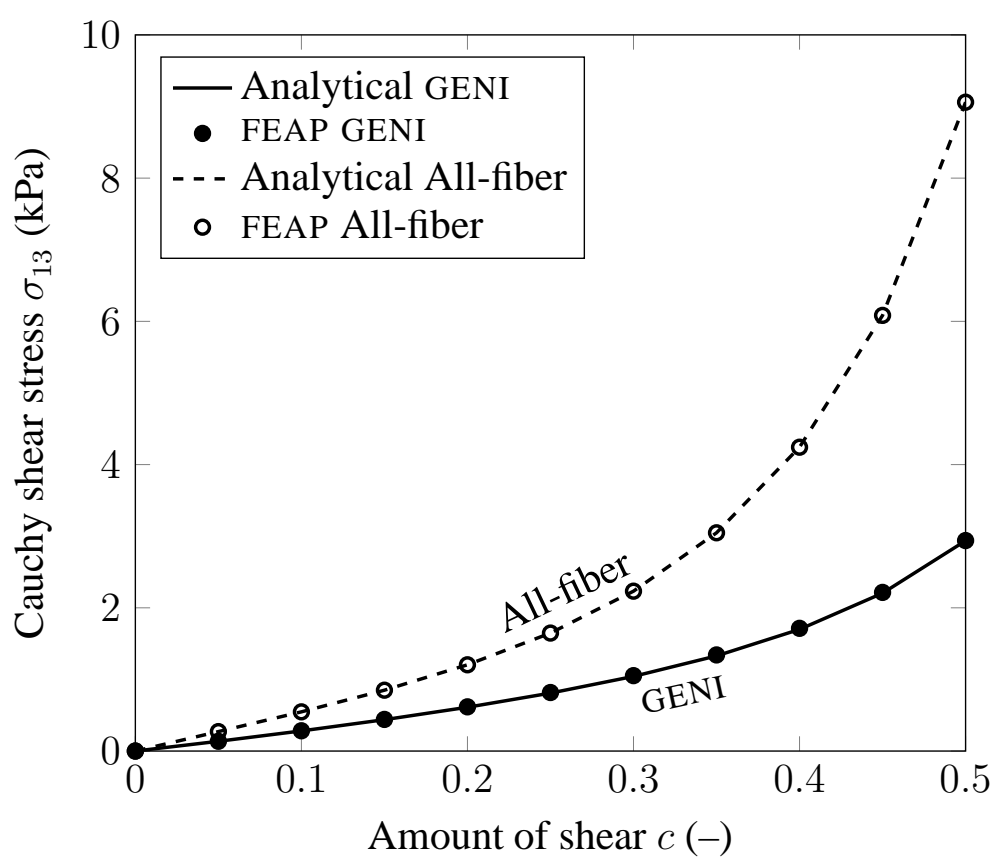

Figure 5: Comparison of the computational results with the analytical solution (49) for a simple shear test of a unit cube with parameters $\mu=2.0 \mathrm{kPa}, k_{1}=10.0 \mathrm{kPa}, k_{2}=25$, and $b=1.0$. The solid curve and dots represent the material response with compressed fibers excluded (GENI model); the dashed curve and open circles are the results for the case with all fibers included (All-fiber model).

NIntegrate function and the Boole operation for the evaluation of the integrals in (48) on the domain defined by (46). The computational results (solid dots) obtained by using FEAP [29] and the analytical solution (solid curve) obtained by using MATHEMATICA [35] for $\sigma_{13}$ versus $c$ are plotted in Figure 5, with the material parameters $\mu=2.0 \mathrm{kPa}, k_{1}=10.0 \mathrm{kPa}, k_{2}=25$, and $b=1.0$. Again, a very good agreement is observed between the two solutions. For comparison, we also plotted the computational and analytical solutions with the All-fiber model. Due to the assumed alignment of the mean fiber direction, most of the fibers within the dispersion are under compression, similarly to the results of Figure 7 in [16]. By excluding the contributions from the compressed fibers in the strain-energy function, a significantly lower shear stress response is observed with the GENI model than with the All-fiber model.

\subsection{Unconfined compression of articular cartilage}

To further illustrate the influence of excluding fibers under compression on the mechanical response, we now consider an unconfined compression test on a circular cylindrical specimen of 
articular cartilage. The effect of excluding compressed fibers becomes most pronounced when the cartilage is compressed in the thickness direction, in which a large proportion of the fibers are aligned. In this example, the purpose is not to create a sophisticated model of cartilage tissue but rather to use a simplified model to demonstrate the performance of the proposed model in a larger scale simulation.

The mechanical properties and durability of cartilage tissue depend primarily on its highly organized collagen fiber network [36]. Through the thickness direction, the modeling is often divided into three zones: superficial, middle, and deep zones. Due to the low fluid permeability of cartilage tissue, it is not easy to squeeze the water out. Hence, cartilage behaves mechanically as a single-phase solid for short static loading periods or for cyclic loading with moderate or high frequencies [37]. Thus, in this example, we treat cartilage tissue simply as a nonlinear, incompressible, single-phase, and multi-zonal fibrous tissue. The viscoelastic behavior is not considered in this study. It is well established that the collagen fibers are distributed in the three zones of mature cartilage as follows: (i) in the superficial zone (SZ) the fibers are oriented tangentially to the articular surface; (ii) in the middle zone (MZ) the fibers have no predominant orientation and are thus randomly distributed; (iii) in the deep zone (DZ) the fibers become aligned perpendicularly to the articular surface and the bone-cartilage interface (see the image analysis in [36]).

Due to the lack of sufficient zone-specific mechanical data for cartilage, we estimated the material parameters of the cartilage by using the depth averaged compression data of cartilage [38]. Specifically, because of the fiber alignment in the cartilage, it is assumed that the depth averaged Cauchy stress versus stretch response of the full-thickness bovine cartilage represents approximately the behavior of the middle zone. This is also motivated by experimental results which show that the mechanical response of the middle zone is closest to that of the full-thickness specimen [39]. We then fit the proposed model to the unconfined compression test data of bovine femoral cartilage [38] at a loading frequency of $1 \mathrm{~Hz}$ and obtained depth averaged material parameters of the MZ. For the SZ and DZ, we adopted the same material parameters, namely $\mu, k_{1}, k_{2}$, but we have used different structural parameters. Note that it is not sensible to fit the overall stress-stretch data of the full-thickness cartilage to the analytically computed Cauchy stress separately for each of the three zones.

For the model fitting, we can still use the analytical Cauchy stress (43) from Section 4.1 because the fibers in the $\mathrm{MZ}$ are dispersed uniformly $(b=0$ and $\rho(\Theta, \Phi)=1)$. The integrations 


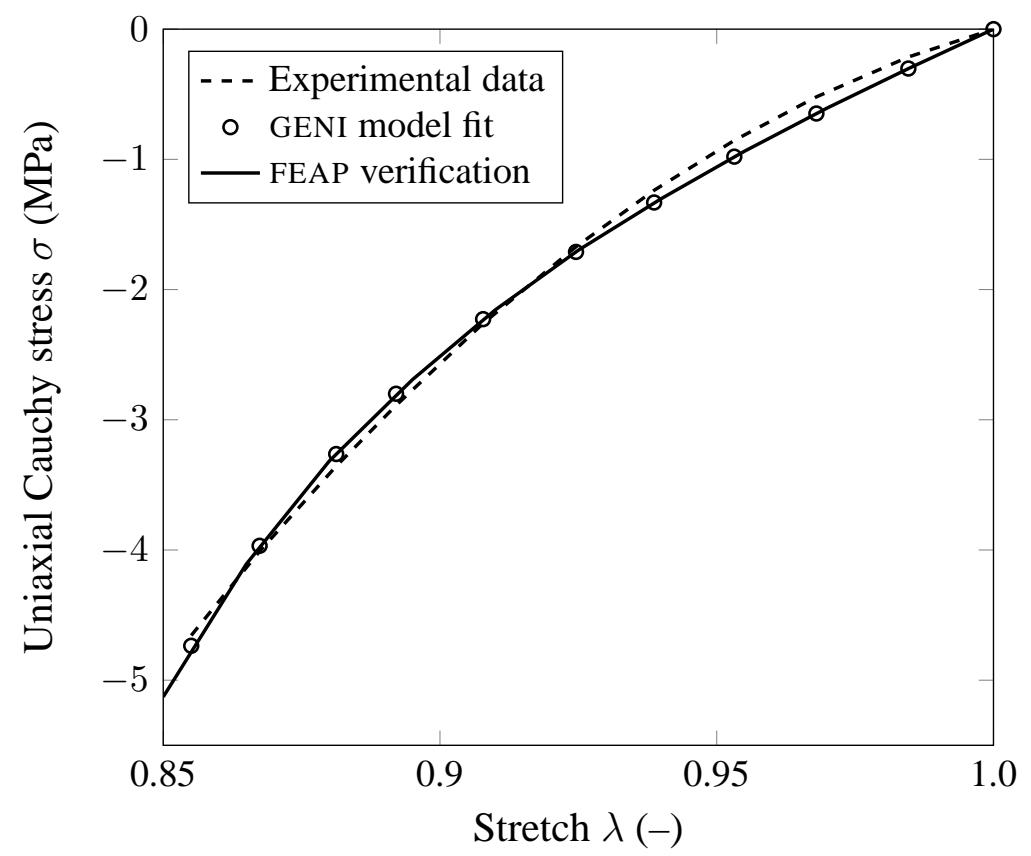

Figure 6: Comparison of the GENI model fit (open circle) and the experimental data (dashed curve) for an unconfined compression test on bovine articular cartilage [38]. Also shown is the FEAP verification (solid curve) of the compression test by using the fitted parameters of Table 1 . Note that the FEAP analysis was performed with a stretch of 0.85 .

in (37) now become

$$
s_{1}=\int_{\Theta_{c}}^{\pi / 2} \sin \Theta \mathrm{d} \Theta, \quad s_{3}=\int_{\Theta_{c}}^{\pi / 2} \sin ^{3} \Theta \mathrm{d} \Theta, \quad s_{5}=\int_{\Theta_{c}}^{\pi / 2} \sin ^{5} \Theta \mathrm{d} \Theta,
$$

which can be evaluated exactly, but we omit the explicit expressions here. On substituting these into $\alpha$ and $\beta$, i.e. (41) and (42) $)_{2}$, we obtain the stress component (43) in the thickness direction as a function of stretch. We then fitted this result to the experimental data and obtained the material parameters $\mu=2.70 \mathrm{MPa}, k_{1}=34.69 \mathrm{MPa}, k_{2}=43.12$, and $b=0$, with the coefficient of determination $r^{2}=0.99$. For comparison, the experimentally measured and analytically computed stress-stretch curves are plotted in Figure 6 together with the results obtained by using FEAP [29] with the fitted parameters and the unit cube model, as described in Section 4.1. As can be seen, we obtain very good agreement between the fitted result and the FEAP solution. This set of material parameters is then applied to all three zones separately. However, the concentration parameter $b$ is different for each of the zones, and the mean fiber directions in the SZ and the DZ are different. Since the fibers are highly aligned in the SZ, we could use the HGO model [25]. But to fully test the performance of the proposed GENI model, we employed it in each of the three zones. We adopted the concentration parameters 
Table 1: Material and structural parameters of the cartilage model [38].

\begin{tabular}{c|c|c|c|c|c|c}
\hline Model & Zone & $\mu(\mathrm{MPa})$ & $k_{1}(\mathrm{MPa})$ & $k_{2}(-)$ & $b(-)$ & $\Theta_{\mathrm{M}}\left({ }^{\circ}\right)$ \\
\hline \multirow{3}{*}{ GENI } & Superficial & & & & 5.0 & 0 \\
& Middle & 2.70 & 34.69 & 43.12 & 0.0 & - \\
& Deep & & & & 2.0 & 90 \\
\hline \multirow{3}{*}{ All-fiber } & Superficial & & & & 5.0 & 0 \\
& Middle & 2.70 & 15.80 & 41.30 & 0.0 & - \\
& Deep & & & & 2.0 & 90 \\
\hline
\end{tabular}

$b=5$ for the SZ, $b=0$ for the MZ, and $b=2$ for the DZ [40], as summarized in Table 1 . Similarly, we have also fitted the All-fiber model to the same experimental data and obtained another set of material parameters, see Table 1. The analytical solution of the All-fiber model is obtained by replacing $\Theta_{c}$ with 0 in (50). Because the difference of the two models lies in the fiber contribution, the shear modulus $\mu$ stays the same. This set of material parameters has also been verified by using the unit cube model. Again, we obtained a very good match between the fitted result and the FEAP solution (not shown here). The mean fiber direction in the DZ is aligned with the thickness direction $\left(90^{\circ}\right.$ measured from the articular surface), while in the SZ it is aligned tangentially to the articular surface.

Based on the dimensions of the specimen in the experimental study [38], we have created a cartilage model of $3.0 \mathrm{~mm}$ in diameter and $1.83 \mathrm{~mm}$ in thickness. The total thickness of the cartilage is supposed to be $2.33 \mathrm{~mm}$. However, a $0.5 \mathrm{~mm}$ thick layer of tissue was removed from the DZ in the experiment. We then obtained the ratio of the thicknesses in each zone of bovine femoral cartilage from Figure 7 of [41]. With these data, after subtraction of $0.5 \mathrm{~mm}$ from the DZ, we obtained the thickness of each zone as $0.42,0.56$, and $0.85 \mathrm{~mm}$ for SZ, MZ, and DZ, respectively, as indicated in Figure 7(a). Due to the symmetry of the specimen and the fiber distribution, we only simulated one half of the specimen. The geometry was then discretized with 1430 8-node hexahedral elements in ABAQUS/CAE [21] and depicted in Figure 7(b).

The generated mesh file was then converted into the input file format of FEAP [29]. For the unconfined compression simulation, all the nodes on the bottom face of the model were constrained in the $\mathbf{E}_{3}$ direction. All the nodes on the symmetry plane, which is the $\left(\mathbf{E}_{1}, \mathbf{E}_{3}\right)$ plane, were constrained in the $\mathbf{E}_{2}$ direction. Furthermore, to prevent rigid body motion, we constrained the $\mathbf{E}_{1}$ direction degree of freedom at the center node of the bottom surface shown as the red dot in Figure 7 (b). A displacement of $-0.23 \mathrm{~mm}$, which was determined from the 
(a)

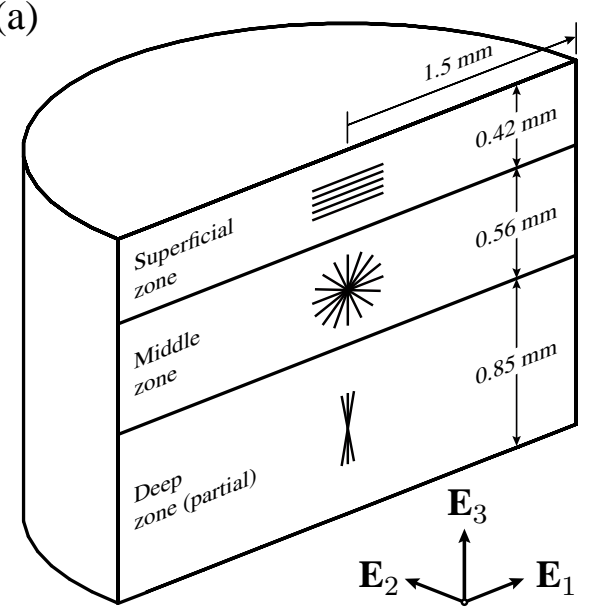

(c)

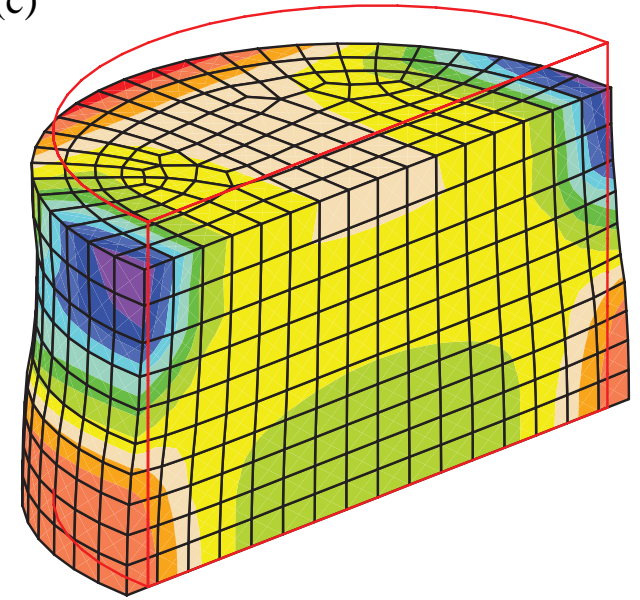

(b)
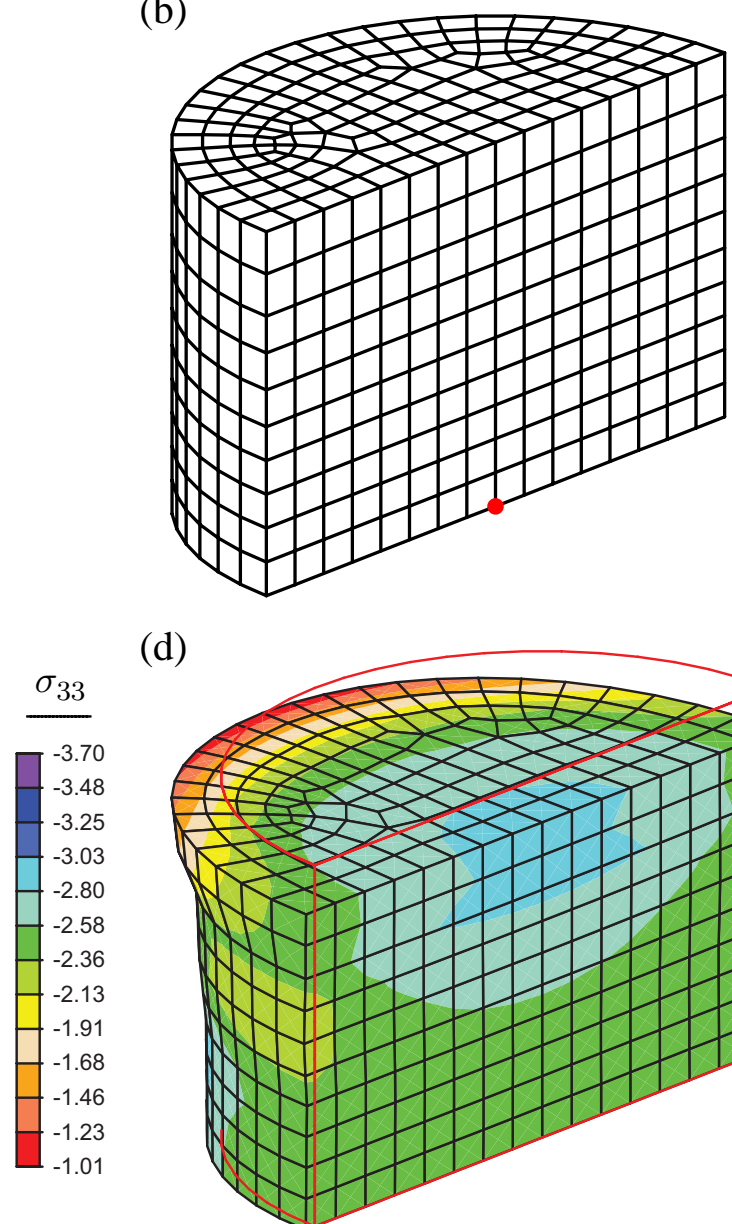

(d)

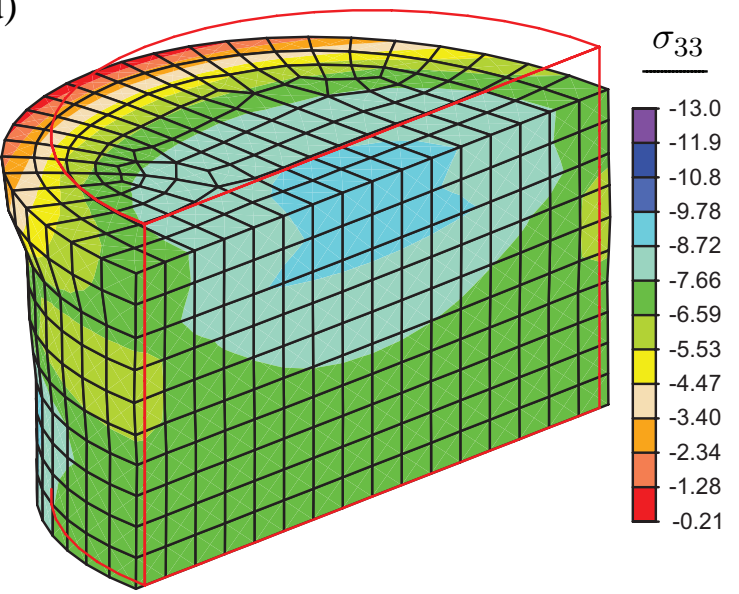

(e)

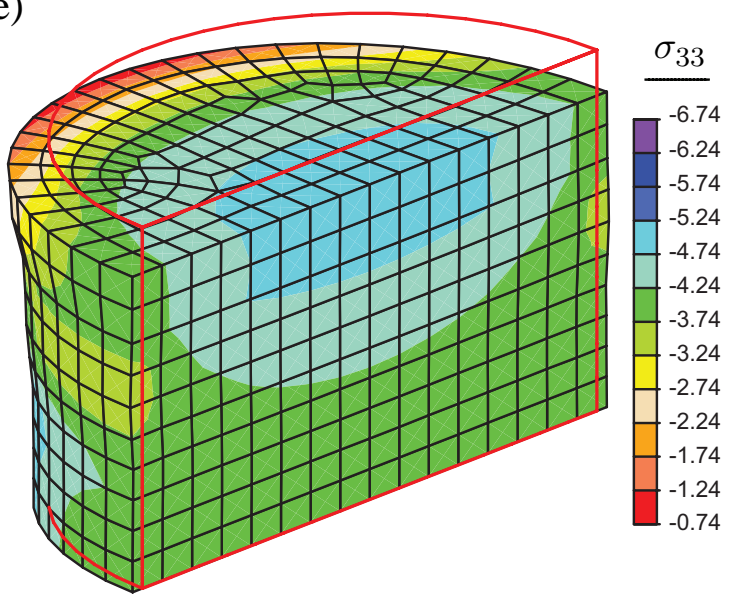

Figure 7: Unconfined compression simulation of articular cartilage up to a stretch of 0.87 : (a) geometry of the cartilage model (one half of the specimen); (b) finite element mesh with 1430 hexahedral elements; (c) deformed configuration showing the normal Cauchy stress distribution $\sigma_{33}$ (in $\mathrm{MPa}$ ) with the GENI model; (d) deformed configuration showing the normal Cauchy stress distribution with the All-fiber model and GENI model parameters; (e) deformed configuration showing the normal Cauchy stress distribution with All-fiber model and its own material parameters (Table 1). The red frames in (c), (d), and (e) indicate the initial configurations. 
experiment [38], was prescribed on the top face of the model for the compression test.

The finite element analysis of the cartilage model was performed with FEAP [29]. The simulation results reveal a non-homogeneous Cauchy stress distribution $\left(\sigma_{33}\right)$ in the configuration at a stretch of 0.87 , as shown in Figure 7(c). As expected, a very small displacement is observed in the $\mathbf{E}_{1}$ direction in the SZ because of the highly concentrated fibers in that direction, while the displacement in the $\mathbf{E}_{2}$ direction is the largest of all three zones because of the particular fiber alignment. The displacement in the $\mathrm{MZ}$ is, in general, larger than in the $\mathrm{SZ}$ in the $\mathbf{E}_{1}$ direction, but smaller than for the SZ in the $\mathbf{E}_{2}$ direction. In the $\mathrm{DZ}$, because of the exclusion of compressed fibers in the $\mathbf{E}_{3}$ direction, the displacement reaches its peak in the $\mathbf{E}_{1}$ direction, and close to the peak in the $\mathbf{E}_{2}$ direction observed in the SZ. Thus, we conclude that the displacement increases nonlinearly in the $\mathbf{E}_{1}$ direction, but in the $\mathbf{E}_{2}$ direction the displacement is smallest in the MZ. We found that the displacement pattern for the three zones in the $\mathbf{E}_{1}$ direction is very similar to that of the simulation results for a multiphasic, axisymmetric cartilage model in [41].

For comparison we have also performed a finite element analysis for the same cartilage model without the exclusion of compressed fibers (All-fiber model with GENI model parameters). This again results in a non-homogeneous Cauchy stress distribution $\left(\sigma_{33}\right)$, as shown in Figure 7(d). As can be seen, the displacements are somewhat different from those of the GENI model shown in Figure 7(c). Indeed, the peak value of the displacement in the $\mathbf{E}_{2}$ direction observed in the SZ is about $121 \%$ larger than that of the GENI model. The simulation results indicate that the displacement in the $\mathbf{E}_{1}$ direction has a saddle shape with that in the MZ being the largest, see Figure 7(d), and in the $\mathbf{E}_{2}$ direction decreases nonlinearly from the SZ to the DZ. Thus, they are completely different from the GENI model predictions. Interestingly, the deformation pattern in the $\mathbf{E}_{1}$ direction, when compressed fibers are not excluded, is similar to the corrected simulation results described in [42], in which the compressed fibers were also not excluded.

For a further comparison, we carried out another finite element analysis of the same cartilage model by using the All-fiber model with its own model parameters (Table 1), see Figure 7(e). Hence, we are testing the capability of the All-fiber model in modeling of the cartilage under compression. Again, we observed very similar deformation pattern and stress distribution with respect to the case when the All-fiber model is used with the GENI model parameters (Figure 7(d)). The only major difference is the magnitude of the stress due to the smaller values of the material parameters $k_{1}$ and $k_{2}$. Note that, in general, the All-fiber model should not be used for modeling of fibrous tissues. 


\section{Concluding remarks}

Based on a new general invariant, we have proposed an exponential fiber dispersion model capable of excluding fibers under compression in the modeling of the highly nonlinear response of fibrous soft biological tissues. We have derived expressions for the associated Cauchy stress and elasticity tensors and have implemented the model in a finite element analysis program. The model has been tested with three numerical examples. We have observed very good agreement with the analytical solutions in the first two examples. Because of the complex nonhomogeneous deformation in the last example, an analytical solution is not available for comparison with the simulation results. We have also performed finite element analyses for the three examples using the All-fiber model and found that the simulation results are completely different from those for the GENI model. Exclusion of compressed fibers in a fiber dispersion model plays an important role in the modeling of fibrous tissues. Besides the capability of excluding fibers under compression, the other advantage of the proposed GENI model is that it requires less computational time in a way similar to the GST approach [17], because the total contribution of fibers under tension is 'wrapped up' into a scalar invariant. This invariant is then used in the exponential strain-energy function. This method is faster than the one which uses an exponential function in the integrand of the strain-energy function according to [16].

In general, if under some loading conditions the contribution of the compressed fibers to the total strain-energy function is much smaller than that of the fibers under tension, then the exclusion of compressed fibers may have a very small influence on the overall material behavior. In that case, the mechanical response of the fiber dispersion model with the compressed fibers excluded is very similar to that of the All-fiber model. However, in scenarios where the contribution of the compressed fibers to the total strain energy is relatively large, then the model which excludes the compressed fibers should be adopted. Thus, it really depends on how the fibers are distributed within a dispersion in space and how many of them are compressed when choosing an appropriate constitutive model for fibrous tissues.

In the third numerical example, we found that the lateral displacements of the cartilage tissue under unconfined compression are in general large in the DZ when the GENI model is used. Because the DZ is connected to the bone underneath this may restrict the lateral displacement, especially in the DZ itself, but this is not considered in the present study. Because of the complex deformation pattern under unconfined compression, the axisymmetric finite element model [41] is not sufficient for investigating the deformation of cartilage. Thus, a 3D model for simulating the cartilage response with dispersed fibers should be adopted.

In the present study, we have adopted the quadratic form of $f\left(\bar{I}_{4}\right)$ for a single fiber given in 
(27). It is straightforward to use a more general form of $f\left(\bar{I}_{4}\right)$ if required. We also note that collagen fibers may be distributed more densely in some biological tissues, in which case the volume fraction of the fibers could be included in the model and (15) replaced by

$$
\Psi_{\text {iso }}=(1-\eta) \Psi_{\mathrm{g}}\left(\bar{I}_{1}\right)+\eta \Psi_{\mathrm{f}}(\bar{I})
$$

where $\eta$ represents the layer-specific volume fraction of the fibers in the material. Finally, in our study, we have adopted a 3D fiber dispersion around a mean direction. We note, however, that for some tissues a planar fiber dispersion would be more appropriate, as exemplified in [15]. Moreover, a rotationally symmetric fiber dispersion has been assumed in all three numerical examples, although the general framework of Section 3 is not restricted to rotationally symmetric dispersions. An extension to a non-symmetric fiber dispersion, which is more realistic for arterial walls [2], can also be accommodated. Such extensions are beyond the scope of the current study but can be included in future works.

\section{Acknowledgments}

We would like to acknowledge the use of HPC resources provided by the Information Technology Services (ZID) of Graz University of Technology, Austria.

\section{Appendix A: Derivations of $\overline{\mathbf{H}}$ and $\overline{\mathbb{H}}$ for an incompressible ma- terial}

In this appendix we consider the incompressible specialization in which case the bar can be dropped from $\overline{\mathbf{H}}$ and $\overline{\mathbb{H}}$. Because the boundary of the integration domain $\Omega$ in the definition (4) of the general invariant $I$ depends on the deformation through $\mathbf{C}$, differentiation of $I$ with respect to $\mathbf{C}$ following the Leibniz integral rule for multi-dimensional integrals reads (without the factor $1 /(2 \pi)$ )

$$
\frac{\partial}{\partial \mathbf{C}} \int_{\Omega(\mathbf{C})} F(\mathbf{C}, \Theta, \Phi) \mathrm{d} \Omega=\int_{\Omega(\mathbf{C})} \frac{\partial}{\partial \mathbf{C}} F(\mathbf{C}, \Theta, \Phi) \mathrm{d} \Omega+\int_{\partial \Omega(\mathbf{C})} F(\mathbf{C}, \Theta, \Phi) \mathcal{N} \otimes \mathcal{N} \mathrm{d} s
$$

where $F(\mathbf{C}, \Theta, \Phi)=\rho(\Theta, \Phi) f\left(I_{4}\right), \partial \Omega(\mathbf{C})$, with unit outward normal $\mathcal{N}$, denotes the boundary of $\Omega, \mathrm{d} \Omega=\sin \Theta \mathrm{d} \Theta \mathrm{d} \Phi$, and $\mathrm{d} s$ is a line element on $\partial \Omega(\mathbf{C})$. Because $I_{4}=1$ on the boundary and $f(1)=0, F(\mathbf{C}, \Theta, \Phi)=0$. Thus, the second term on the right-hand side of (52) vanishes and does not appear in (18) in the incompressible limit. 
Similarly to the result in (52), the second derivative of $I$ with respect to $\mathbf{C}$ reads (without the factor $1 /(2 \pi)$ )

$$
\begin{aligned}
\frac{\partial}{\partial \mathbf{C}} \int_{\Omega(\mathbf{C})} \frac{\partial}{\partial \mathbf{C}} F(\mathbf{C}, \Theta, \Phi) \mathrm{d} \Omega= & \int_{\Omega(\mathbf{C})} \frac{\partial^{2}}{\partial \mathbf{C} \partial \mathbf{C}} F(\mathbf{C}, \Theta, \Phi) \mathrm{d} \Omega \\
& +\int_{\partial \Omega(\mathbf{C})} \frac{\partial}{\partial \mathbf{C}} F(\mathbf{C}, \Theta, \Phi) \otimes \mathcal{N} \otimes \mathcal{N} \mathrm{d} s .
\end{aligned}
$$

Again, because on the boundary $I_{4}=1$ and $f^{\prime}(1)=0$, the second term on the right-hand side of (53) vanishes and does not appear in (23) in the incompressible limit.

\section{References}

[1] G. A. Holzapfel and R. W. Ogden. Constitutive modelling of arteries. Proc. R. Soc. Lond. A, 466:1551-1597, 2010.

[2] G. A. Holzapfel, J. A. Niestrawska, R. W. Ogden, A. J. Reinisch, and A. J. Schriefl. Modelling non-symmetric collagen fibre dispersion in arterial walls. J. R. Soc. Interface, 12:20150188, 2015.

[3] W. Mao, K. Li, and W. Sun. Fluid-structure interaction study of transcatheter aortic valve dynamics using smoothed particle hydrodynamics. Cardiovasc. Eng. Technol., 7:374-388, 2016.

[4] A. J. Schriefl, G. Zeindlinger, D. M. Pierce, P. Regitnig, and G. A. Holzapfel. Determination of the layer-specific distributed collagen fiber orientations in human thoracic and abdominal aortas and common iliac arteries. J. R. Soc. Interface, 9:1275-1286, 2012.

[5] A. J. Schriefl, H. Wolinski, P. Regitnig, S. D. Kohlwein, and G. A. Holzapfel. An automated approach for 3D quantification of fibrillar structures in optically cleared soft biological tissues. J. R. Soc. Interface, 10:20120760, 2013.

[6] J. A. Niestrawska, Ch. Viertler, P. Regitnig, T. U. Cohnert, G. Sommer, and G. A. Holzapfel. Microstructure and mechanics of healthy and aneurysmatic abdominal aortas: experimental analysis and modeling. J. R. Soc. Interface, 13:20160620, 2016.

[7] M. B. Lilledahl, D. M. Pierce, T. Ricken, G. A. Holzapfel, and C. de Lange Davies. Structural analysis of articular cartilage using multiphoton microscopy: input for biomechanical modeling. IEEE Trans. Med. Imaging, 30:1635-1648, 2011. 
[8] G. A. Ateshian, V. Rajan, N. O. Chahine, C. E. Canal, and C. T. Hung. Modeling the matrix of articular cartilage using a continuous fiber angular distribution predicts many observed phenomena. J. Biomech. Eng., 131:61003, 2009.

[9] L. Azinfar, M. Ravanfar, Y. Wang, K. Zhang, D. Duan, and G. Yao. High resolution imaging of the fibrous microstructure in bovine common carotid artery using optical polarization tractography. J. Biophotonics, 10:231-241, 2017.

[10] W. J. Karlon, J. W. Covell, A. D. McCulloch, J. J. Hunter, and J. H. Omens. Automated measurement of myofiber disarray in transgenic mice with ventricular expression of ras. Anat. Rec., 252:612-625, 1998.

[11] J. W. Covell. Tissue structure and ventricular wall mechanics. Circulation, 118:699-701, 2008.

[12] M. S. Sacks. Biomechanics of native and engineered heart valve tissues. In F. Guilak, D. L. Butler, St. A. Goldstein, and D. J. Mooney, editors, Functional Tissue Engineering, pages 243-257, New York, 2003. Springer-Verlag.

[13] Y. Lanir. Constitutive equations for fibrous connective tissues. J. Biomech., 16:1-12, 1983.

[14] G. A. Holzapfel and R. W. Ogden. On the tension-compression switch in soft fibrous solids. Eur. J. Mech. A/Solids, 49:561-569, 2015.

[15] K. Li, R. W. Ogden, and G. A. Holzapfel. Computational method for excluding fibers under compression in modeling soft fibrous solids. Eur. J. Mech. A/Solids, 57:178-193, 2016.

[16] K. Li, R. W. Ogden, and G. A. Holzapfel. An exponential constitutive model excluding fibers under compression: application to extension-inflation of a residually stressed carotid artery. Math. Mech. Solids. in press.

[17] T. C. Gasser, R. W. Ogden, and G. A. Holzapfel. Hyperelastic modelling of arterial layers with distributed collagen fibre orientations. J. R. Soc. Interface, 3:15-35, 2006.

[18] G. A. Holzapfel and R. W. Ogden. Comparison of two model frameworks for fiber dispersion in the elasticity of soft biological tissues. Eur. J. Mech. A/Solids, 66:193-200, 2017. 
[19] S. G. Advani and C. L. Tucker III. The use of tensors to describe and predict fiber orientation in short fiber composites. J. Rheol., 31:751-784, 1987.

[20] G. A. Holzapfel. Nonlinear Solid Mechanics. A Continuum Approach for Engineering. John Wiley \& Sons, Chichester, 2000.

[21] Dassault Systèmes Simulia Corporation. Abaqus Analysis Guide. Johnston, Rhode Island, United States, 2017.

[22] M. Latorre and F. J. Montáns. On the tension-compression switch of the Gasser-OgdenHolzapfel model: Analysis and a new pre-integrated proposal. J. Mech. Behav. Biomed. Mater., 57:175-189, 2016.

[23] G. A. Holzapfel and R. W. Ogden. On fiber dispersion models: exclusion of compressed fibers and spurious model comparisons. J. Elasticity. in press.

[24] H. Weisbecker, C. Viertler, D. M. Pierce, and G. A. Holzapfel. The role of elastin and collagen in the softening behavior of the human thoracic aortic media. J. Biomech., 46: 1859-1865, 2013.

[25] G. A. Holzapfel, T. C. Gasser, and R. W. Ogden. A new constitutive framework for arterial wall mechanics and a comparative study of material models. J. Elasticity, 61:1-48, 2000.

[26] P. N. Watton, Y. Ventikos, and G. A. Holzapfel. Modelling the mechanical response of elastin for arterial tissue. J. Biomech., 42:1320-1325, 2009.

[27] P. J. Flory. Thermodynamic relations for highly elastic materials. Trans. Faraday Soc., 57:829-838, 1961.

[28] R. W. Ogden. Nearly isochoric elastic deformations: Application to rubberlike solids. $J$. Mech. Phys. Solids, 26:37-57, 1978.

[29] R. L. Taylor. FEAP - A Finite Element Analysis Program, Version 8.4 User Manual. University of California at Berkeley, Berkeley, California, 2013.

[30] J. Berntsen, T. O. Espelid, and A. Genz. An adaptive algorithm for the approximate calculation of multiple integrals. ACM Trans. Math. Software, 17:437-451, 1991.

[31] J. Berntsen, T. O. Espelid, and A. Genz. An adaptive multidimensional integration routine for a vector of integrals. ACM Trans. Math. Software, 17:452-456, 1991. 
[32] H. Xiao and Z. Gimbutas. A numerical algorithm for the construction of efficient quadrature rules in two and higher dimensions. Comput. Math. Appl., 59:663-676, 2010.

[33] J. C. Simo and R. L. Taylor. Quasi-incompressible finite elasticity in principal stretches. Continuum basis and numerical algorithms. Comput. Meth. Appl. Mech. Eng., 85:273$310,1991$.

[34] MATLAB. R2016a, The MathWorks Inc., Natick, MA, USA, 2016.

[35] Wolfram Research, Inc. Mathematica, Version 10.4. Champaign, Illinois, 2016.

[36] A. Changoor, M. Nelea, S. Méthot, N. Tran-Khanh, A. Chevrier, A. Restrepo, M. S. Shive, C. D. Hoemann, and M. D. Buschmann. Structural characteristics of the collagen network in human normal, degraded and repair articular cartilages observed in polarized light and scanning electron microscopies. Osteoarthr. Cartil., 19:1458-1468, 2011.

[37] D. R. Carter and M. Wong. Modelling cartilage mechanobiology. Philos. T. Roy. Soc. B, 358:1461-1471, 2003.

[38] S. Park, C. T. Hung, and G. A. Ateshian. Mechanical response of bovine articular cartilage under dynamic unconfined compression loading at physiological stress levels. Osteoarthr. Cartil., 12:65-73, 2004.

[39] A. C. Chen, W. C. Bae, R. M. Schinagl, and R. L. Sah. Depth- and strain-dependent mechanical and electromechanical properties of full-thickness bovine articular cartilage in confined compression. J. Biomech., 34:1-12, 2001.

[40] S. Federico and T. C. Gasser. Nonlinear elasticity of biological tissues with statistical fibre orientation. J. R. Soc. Interface, 7:955-966, 2010.

[41] P. Julkunen, P. Kiviranta, W. Wilson, J. S. Jurvelin, and R. K. Korhonen. Characterization of articular cartilage by combining microscopic analysis with a fibril-reinforced finiteelement model. J. Biomech., 40:1862-1870, 2007.

[42] S. Federico, J. Pajerski, and T. C. Gasser. Corrigendum for the paper 'nonlinear elasticity of biological tissues with statistical fibre orientation'. J. R. Soc. Interface, 8:1367-1368, 2011. 